\title{
Exploiting architectural features of a computer vision platform towards reducing memory stalls
}

\author{
Naveed UI Mustafa ${ }^{1}$ (D) Martin J. O'Riordan ${ }^{2} \cdot$ Stephen Rogers ${ }^{2} \cdot$ Ozcan Ozturk $^{1}$
}

Received: 24 September 2017 / Accepted: 1 October 2018 / Published online: 9 October 2018

c) Springer-Verlag GmbH Germany, part of Springer Nature 2018

\begin{abstract}
Computer vision applications are becoming more and more popular in embedded systems such as drones, robots, tablets, and mobile devices. These applications are both compute and memory intensive, with memory bound stalls (MBS) making a significant part of their execution time. For maximum reduction in memory stalls, compilers need to consider architectural details of a platform and utilize its hardware components efficiently. In this paper, we propose a compiler optimization for a vision-processing system through classification of memory references to reduce MBS. As the proposed optimization is based on the architectural features of a specific platform, i.e., Myriad 2, it can only be applied to other platforms having similar architectural features. The optimization consists of two steps: affinity analysis and affinity-aware instruction scheduling. We suggest two different approaches for affinity analysis, i.e., source code annotation and automated analysis. We use LLVM compiler infrastructure for implementation of the proposed optimization. Application of annotation-based approach on a memory-intensive program shows a reduction in stall cycles by $67.44 \%$, leading to $25.61 \%$ improvement in execution time. We use 11 different image-processing benchmarks for evaluation of automated analysis approach. Experimental results show that classification of memory references reduces stall cycles, on average, by $69.83 \%$. As all benchmarks are both compute and memory intensive, we achieve improvement in execution time by up to $30 \%$, with a modest average of $5.79 \%$.
\end{abstract}

Keywords Computer vision $\cdot$ Compiler optimization $\cdot$ Execution time $\cdot$ Memory bound stalls

\section{Introduction}

Computer vision (CV) is a rapidly growing field, mostly devoted to capturing, analysis, modification, and understanding of images [1,2]. With the arrival of high-resolution cameras in mobile devices, CV applications are becoming more popular [1]. Embedded systems such as wearable devices, drones, robots, and tablets are supposed to support $\mathrm{CV}$

Naveed Ul Mustafa

naveed.mustafa@bilkent.edu.tr

Martin J. O’Riordan

martin.oriordan@movidius.com

Stephen Rogers

stephen.rogers@movidius.com

Ozcan Ozturk

ozturk@cs.bilkent.edu.tr

1 Department of Computer Engineering, Bilkent University, Ankara, Turkey

2 Movidius-Intel, Dublin, Ireland applications [3]. Domains that employ CV include surveillance $[4,5]$, gesture recognition [6], face tracking [7, 8], medical imaging $[9,10]$, automotive safety $[11,12]$, and food industry [13-15], among others.

Computer vision applications are computationally expensive and mostly required to execute in real time [1]. However, embedded platforms are limited on the power budget. There are two architectural solutions to reduce the power consumption and running the $\mathrm{CV}$ algorithms faster on embedded systems. One popular approach is to use a multicore platform. In general, two smaller cores collectively occupying the same area and consuming the same energy as compared to a single large core can provide $70-80 \%$ higher performance [16]. The other possible approach is using the dedicated optimized cores to implement the commonly used algorithms. This can be achieved using domain-specific hardware accelerators [1]. Besides employing architectural solutions, it is critical for a compiler to reduce the execution time of applications by taking into account the architectural features of the hardware platform [17]. 
There have been various efforts to design vision-processing systems targeting CV applications such as [2, 17-19], among others. One such effort is Myriad 2 platform from Movidius [20]. It is a low-power multi-processor system on chip (MPSoC) that uses an array of very long instruction word (VLIW) processors with vector and single instruction multiple data (SIMD) execution capabilities [21]. Each processor supports two load and store units (LSUs) to overlap latency of memory operations. Since CV applications are heavy in both computation and memory requirements [22], the platform features a high bandwidth memory subsystem. However, being unaware of the memory organization, the compiler of Myriad 2 platform schedules memory accesses inefficiently. This results in unnecessary memory stalls and hence higher execution time for applications.

In this paper, we motivate the need to reduce memory bound stalls (MBS) in CV applications and identify the problem faced by the compiler of Myriad 2 platform in reducing such stalls. Our main contributions in this paper can be summarized as follows.

1. We propose an optimization through classification of memory references aiming to reduce MBS. The optimization consists of two steps: affinity analysis and affinity-aware instruction scheduling (AAIS). While affinity analysis predicts the physical memory location for each memory object in the application's source code, AAIS generates a stall-saving instruction schedule based on the results of affinity analysis step. A compiler equipped with the proposed optimization is named an affinityaware compiler (AAC).

2. We propose two different affinity analysis approaches along with their motivation, namely, source code annotation and automated analysis.

The proposed optimization is based on efficiently utilizing the hardware components of Myriad 2 and, therefore, not applicable as it is to other platforms. However, it is expected to be relatively easy to adapt the optimization for other CV platforms with similar architectural features.

We implement the proposed optimization on LLVM compiler infrastructure [23] and evaluate it by running benchmarks on the Myriad 2 board using the base compiler (BC) and the AAC. We apply annotation-based analysis approach only on a simple memory-intensive test program. It shows the reduction in stall cycles by $67.44 \%$ resulting in $25.61 \%$ improvement in the execution time. We evaluate the automated analysis approach by running 11 different compute and memory-intensive image-processing benchmarks on a Myriad 2 board using the AAC. Results show that AAC reduces stall cycles by $69.83 \%$ with a modest improvement in the execution time by $5.79 \%$, on average, as compared to the $\mathrm{BC}$.
The rest of this paper is organized as follows. Section 2 describes the related work, while Sect. 3 motivates the reduction of MBS for CV applications. Section 4 provides the necessary details of a Myriad 2 platform needed to understand this work. Section 5 formulates the problem faced by the compiler in reducing MBS and proposes the solution. Section 6 provides the implementation details. Methodology for evaluation of proposed optimization is described in Sect. 7, while Sect. 8 shows evaluation results. Section 9 concludes this paper.

\section{Related work}

Memory bound stalls cause underutilization of the compute logic due to memory latency and hence become a major hurdle in improving the execution time of an application [24]. Various approaches have been proposed to reduce memory stalls, such as data mapping in multi-bank environment, using non-uniform memory access (NUMA)-based design and architectural improvements in the memory and compute fabric.

Platforms with multi-bank memory system mitigate the problem by mapping simultaneously requested data on different memory banks. Researchers have presented proposals to implement data mapping as a back-end compiler optimization $[25,26]$ as well as by analyzing memory access pattern at higher levels [27-29] for single-processor systems. Other works, such as [30,31], propose approaches for mapping data of different applications to multiple memory banks in a multi-core environment.

NUMA is commonly used in modern multi-processor systems to avoid the bottleneck of shared memory accesses [32]. It provides asymmetric memory bandwidth and latency characteristics [33]. In other words, cost of accessing data located in remote memory modules is higher than accessing data blocks in local memory modules. Memory affinity is a way to reduce this cost by placing the data in memory modules closer to the processor executing the computation thread [34] and guarantees to improve memory bandwidth and latency [35].

Many researchers have contributed in the context of memory affinity to reduce memory access cost on NUMA platforms. For example, an NUMA API for Linux was proposed in [36] which allows programmers to make memory allocations from a specific node or memory module, in addition to binding threads to specific CPUs. Different algorithms have been proposed to spread application data across different memories of an NUMA platform, such as round-robin, first touch affinity and next-touch affinity $[32,37]$. An extension to Linux kernel to add support for the affinity-on-next-touch algorithm is reported in [38]. 
In this work, we exploit the availability of dual load-store Units to processors of a vision-processing system and its NUMA architecture, where memory is divided into multiple slices, each one having an affinity to one of the processors. Unlike the traditional memory affinity approach focusing on the reduction of latency by placing data closer to the computing processor [32, 33, 37, 39], the purpose of our affinity analysis is to reduce the memory bound stalls by taking into account memory organization and hence efficiently scheduling memory accesses.

Another approach to reducing memory stalls, more related to our work, is optimization of the memory subsystem of the execution platform and related architectural components of the compute fabric. Like Myriad 2 platform, Snapdragon 800 [40], MaPU [17], and TI AcceleratorPac [18] use VLIW processors as main execution units [19] combined with RISC cores and other dedicated components. Unlike these systems using unified memory, Myriad 2 uses NUMA architecture enabling multiple cores to access their local memory slices simultaneously and hence make a contribution in reducing memory stalls.

Hexagon DSP on Snapdragon 800 is a VLIW featuring two data units. Each data unit is capable of executing a load, a store or an ALU instruction but unable to pack two memory accesses with one or more ALU instructions in a single cycle. On the other hand, VLIW processors of Myriad 2 are capable of packing two memory accesses with up to two ALU instructions in a single cycle.

MaPU platform contains ten processing cores with unified memory scheme. A core can make up to three memory accesses simultaneously but into different physical memories. Furthermore, a physical memory cannot be accessed by different cores simultaneously. As compared to MaPU, Myriad 2 supports simultaneous accesses to memory at two levels. First, multiple cores can access their local memory slices simultaneously due to NUMA architecture. Second, each core can make up to two simultaneous accesses into its local slice.

As noted by designers of MapU [17], compilers are a major source of the lower performance of execution platforms as they use a simplified model of processor architecture and do not consider detailed architectural features of the platform. Since our proposed compiler optimization is based on comparatively better architectural features of Myriad 2 platform (such as dual load-store units per processor and a high bandwidth memory subsystem), as shown in Table 1, it is not only different than Hexagon DSP and MaPU, but has a potential of achieving higher performance.

\section{Motivation}

The execution time of an application can be divided into two broad categories: commit cycles and stall cycles. A clock cycle is categorized as a commit cycle if at least one instruction is retired during the cycle; otherwise, it is categorized as a stall cycle. Various reasons such as unavailability of functional units, bad branch prediction, or data dependencies result in stall cycles. The unavailability of data required for instruction execution incurs extra clock cycles in the form of a cache miss penalty. Such cycles are termed memory bound stalls (MBS).

We characterize a set of CV benchmarks to understand the distribution of execution time across different categories. The set consists of benchmarks performing basic image-processing operations such as image addition and subtraction [41], box filtering [42], convolution [43], sum of absolute difference [44], white balancing operation [45], histogram generation, and similarity measurement between pixels of two input images [46]. "Appendix" provides the critical part of the source code for benchmarks. We use Intel's VTune performance analyzer [47] to breakdown the execution time of benchmarks into commit cycles (CC), bad speculation stalls (BSS), MBS, core bound stalls (CBS), and front-end bound stalls (FEBS).

As shown in Fig. 1, on average, MBS make almost 33\% of the total execution time. It suggests the criticality of MBS in reducing the execution time of an application. Therefore, a platform running $\mathrm{CV}$ applications should have an efficient memory architecture supporting the data transactions with high bandwidth and low latency. In addition to that the software infrastructure, such as compiler and assembler, should

Table 1 Comparison of architectural features of different CV platforms

\begin{tabular}{llll}
\hline Platform & $\begin{array}{l}\text { Uses NUMA } \\
\text { architecture }\end{array}$ & $\begin{array}{l}\text { Support for packing more than two memory } \\
\text { instructions with an ALU instruction }\end{array}$ & $\begin{array}{l}\text { Support for multiple simultaneous accesses } \\
\text { by a single processor to the same physical } \\
\text { memory. }\end{array}$ \\
\hline $\begin{array}{l}\text { Snapdragon } 800 \text { (with } \\
\text { Hexagon DSP) }\end{array}$ & $\times$ & $\times$ & $\checkmark$ \\
MaPU & $\times$ & $\checkmark$ & Details not available \\
TI AcceleratorPAC & $\times$ & Details not available & $\checkmark$
\end{tabular}




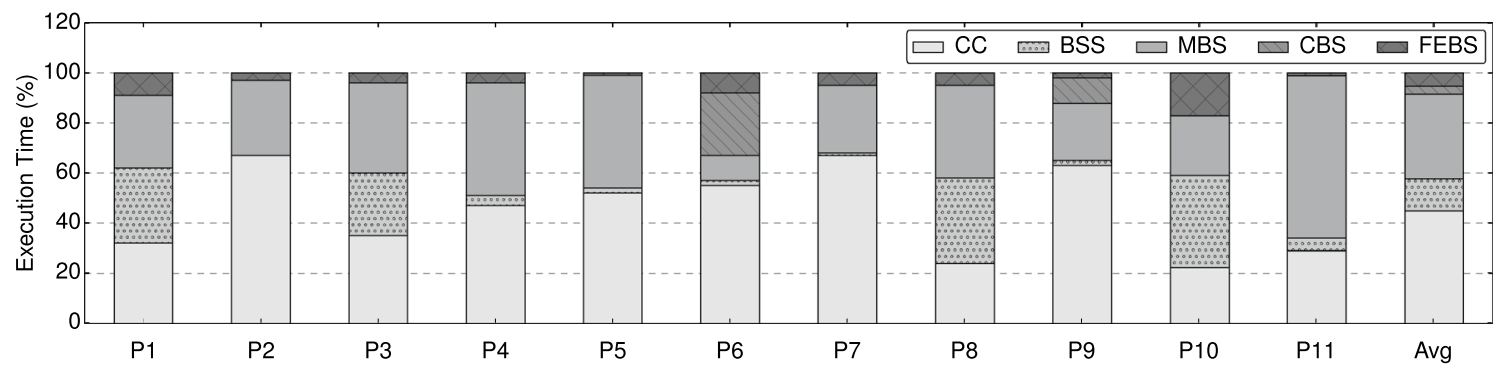

Fig. 1 Benchmarks: P1 = subtraction of two images, P2 = addition of four images, $\mathrm{P} 3$ = addition of two images, $\mathrm{P} 4=$ addition of two images based on a mask input, P5 $=$ box filtering using $5 \times 5$ mask, P6 $=$ addition of two scaled images, $\mathrm{P} 7=$ convolution using $3 \times 3$

take advantage of architectural features offered by the platform to reduce MBS.

\section{Myriad 2 architecture}

Figure 2, based on [48, 49], shows the architectural layout of a Myriad 2 Platform developed by Movidius Ltd [20]. It is an MPSoC containing multiple heterogeneous processors, hardware accelerators, memories, and external interfaces. Target application domain for the Myriad 2 platform is video filtering and image recognition in embedded systems [49]. mask, $\mathrm{P} 8=$ sum of absolute difference using a $5 \times 5$ window, $\mathrm{P} 9=$ white balancing operation, $\mathrm{P} 10=$ histogram generation, and $\mathrm{P} 11=$ similarity measurement between pixels of two images

Myriad 2 contains 12 streaming hybrid architecture vector engine (SHAVE) and two reduced instruction set computing (RISC) processors. SHAVE processors are the real workhorse of Myriad 2 and are designed to crunch the complex imaging and vision algorithms [48]. The platform offers a $2 \mathrm{MB}$ connection matrix (CMX) memory along with a number of programmable hardware accelerators for vision processing. Accelerators are connected to the CMX memory via a crossbar [3].

SHAVE is a VLIW processor containing a set of functional units which are fed with operands from three different register files [21]. The processor contains optimized functional units such as a branch and repeat unit (BRU), a compare and move unit (CMU), arithmetic units, and

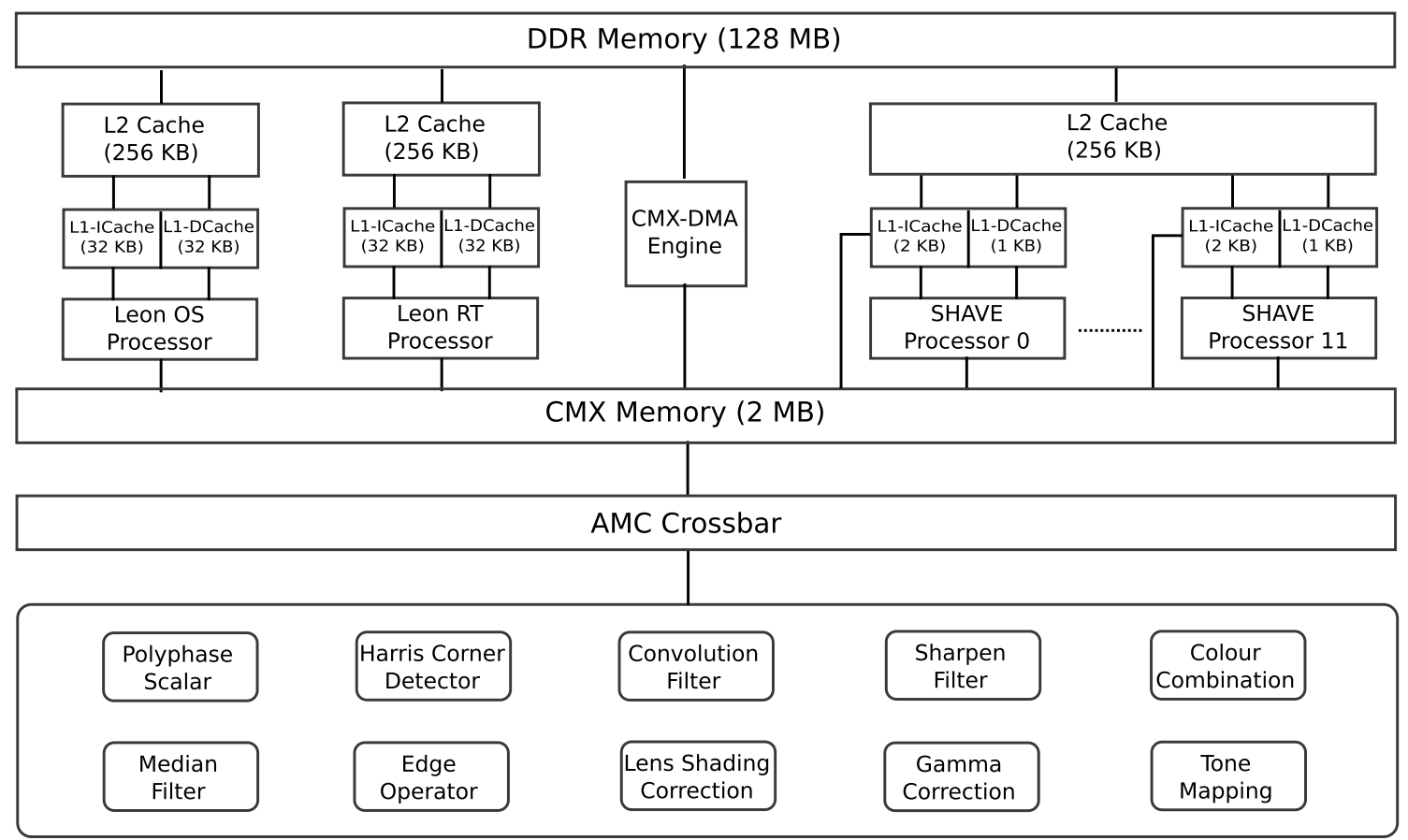

Fig. 2 Architectural layout of a Myriad 2 platform 
two load-store units (LSUs). Each SHAVE processor can execute two load-store instructions simultaneously.

\subsection{CMX memory}

As shown in Fig. 3a, the 2 MB CMX memory is divided into 16 different slices, each with a size of $128 \mathrm{~KB}$. A slice can hold both instructions and the data for a program running on a processor. Each of the first twelve slices (i.e., slice 0 -slice 11) has an affinity to 1 of 12 SHAVE processors. Since Myriad 2 is a non-uniform memory access (NUMA) platform, it is more efficient in terms of latency and energy consumption for a processor to access its local slice (i.e., slice 0 for SHAVE 0). However, processors can also access any other slice in the CMX memory but with a higher latency. Therefore, placement of data in the local slice of a processor is recommended.

A slice is further divided into four regions, named $R 0, R 1, R 2$, and $R 3$ in Fig. $3 \mathrm{~b}$, each with a size of 32 $\mathrm{KB}$. In principle, the architectural design of the CMX memory allows four simultaneous memory accesses in four different regions of a given slice. Each region is a single physical block of random access memory (RAM) with a single chip select and a single set of address and data paths. Therefore, simultaneous memory accesses in the same region are not recommended as they result in stall cycles due to clash among memory ports. Since a SHAVE processor has only two LSUs, only two simultaneous memory accesses are practically possible into a single CMX slice. Simultaneous memory accesses can be performed in any of the two different regions, e.g., $R 0$ and $R 1$ or $R 0$ and $R 2$.

\section{Problem and proposed solution}

In this section, we describe the limitations of a generic compiler in efficiently accessing the CMX memory to reduce the MBS. We also propose a solution to overcome these limitations to generate a stall-saving instruction schedule and hence achieve the faster execution of an application.

\subsection{Problem formulation}

In CV applications, generally, a data frame is processed by applying a filter across all of its pixels. A data frame can occupy a single region of a given slice, multiple regions, or even multiple slices. Since Myriad 2 is an NUMA platform, it is important to place a data frame in a slice local to the computing processor to reduce memory latency.

Another way to reduce memory stalls is to issue multiple simultaneous memory accesses. Since SHAVE is a VLIW processor with two LSUs ( 0 and 1$)$, it is a wastage of resources to perform all memory accesses in a serial fashion. Therefore, the compiler supports scheduling of up to two accesses to a given CMX slice in a single cycle provided that both LSUs are available. However, a check is required to avoid simultaneous accesses in the same region of a given slice due to the clash between memory ports, as described in Sect. 4.1. Since BC does not perform this check and always schedules simultaneously requested memory accesses in the same cycle, it may result in blocking of one memory instruction by another leading to memory stalls.

Such stall cycles can be avoided by making the compiler aware of the architectural limitations of the CMX memory. If provided with the information about the physical location of each memory object in a CMX slice, the compiler can generate optimized instruction schedule leading to the reduction of MBS.
Fig. 3 Organization of the CMX memory and its interface with SHAVE processors in Myriad 2

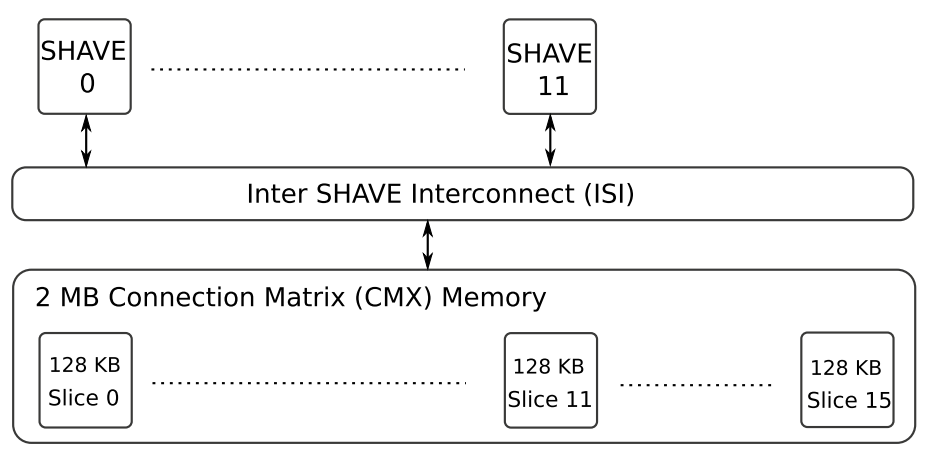

(a) Division of the CMX memory into slices

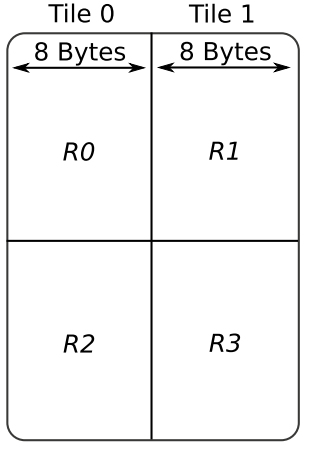

(b) Division of a CMX slice into regions 
It is important to clarify that $\mathrm{BC}$ is already equipped with necessary support needed to resolve conflicts among multiple SHAVE processors and/or accelerators requesting simultaneous accesses to the same memory slice. Therefore, the focus of this work is not the conflict resolution among multiple processors but handling simultaneous accesses by a single processor to the same slice.

\subsection{Proposed solution}

Our proposed solution consists of two steps. In the first step, named affinity analysis, each memory object is appended with an affinity number at compile time to predict its physical location in the CMX slice.

In the second step, i.e., AAIS, the scheduler uses the appended affinity numbers for efficient scheduling of memory instructions. In the AAIS, two instructions requesting simultaneous access in the same CMX slice are scheduled in the same cycle only if two conditions are satisfied:

- Condition 1: Both LSUs are available.

- Condition 2: Memory objects to be accessed by instructions have different affinity numbers.

If any one of these two conditions is not satisfied, instructions are not scheduled in the same cycle. In the rest of this paper, we assume that the first condition is always true.

AAIS reduces stall cycles by avoiding blocking of one memory instruction by another. A compiler using AAIS is named AAC. Otherwise, the scheduling is named basic instruction scheduling (BIS) and the compiler as BC. Note that $\mathrm{BC}$ tests only the first condition for simultaneous scheduling of memory instructions, while AAC tests both of them.

To understand the calculation of affinity numbers, let us divide CMX slice into two logical vertical sections named Tile 0 and Tile1. This logical division is shown in Fig. 3b, where each tile has two physical regions. Let us consider two instruction, Inst 1 and Inst 2 , requesting access in the same
CMX slice simultaneously with addresses for their respective memory objects as $\mathrm{BP}+\Delta_{1}$ and $\mathrm{BP}+\Delta_{2}$, where $B P$ is a base address and $\Delta$ is the offset from BP. Since the base address is not known at compile time, only offset value is to be used to infer the physical location of a memory object.

Assuming that base address is 16-byte aligned (i.e., a multiple of 16), offset can be used to find the ID of the tile a memory object belongs to. Since each tile is 8-byte wide, if the offset address is in the range of $[8 \times n,(8 \times(n+1))-1]$, then the memory belongs to Tile 1 . Otherwise, the memory object belongs to Tile 0 . With $n$ defined as an odd number, the expression $[8 \times n,(8 \times(n+1))-1]$ represents ranges such as $[8, \ldots ., 15],[24, \ldots ., 31]$ and so on. Note that an offset address within these ranges will always have its third bit set to 1 . In other words, using a mask $=0 \times 008$, a memory object with address $B P+\Delta$ belongs to Tile 0 if bitwise and operation between $\Delta$ and mask equals zero and to Tile 1 otherwise.

Depending on the results of masking operations, there are two cases for the calculation of affinity numbers in the affinity analysis step.

- Case A: $\left(\Delta_{1} \&\right.$ mask $) !=\left(\Delta_{2} \&\right.$ mask $)$. In this case, memory objects of Inst 1 and Inst 2 belong to different tiles, guaranteeing that they also belong to different regions, as shown in Fig. 4. Since the AAIS performs a non-equality test on affinity numbers, tile IDs can be appended to memory objects in place of region IDs in the affinity analysis step without the loss of correctness.

As the two memory objects belong to different regions and each region has its own set of memory ports, there is no architectural restriction on simultaneous execution of Inst 1 and Inst2. In this case, both BIS and AAIS generate the same instruction schedule. BIS schedules Instr1 and Instr 2 in the same cycle without testing the second condition. On the other hand, AAIS detects that second condition is true and hence schedules the instructions in the same cycle.

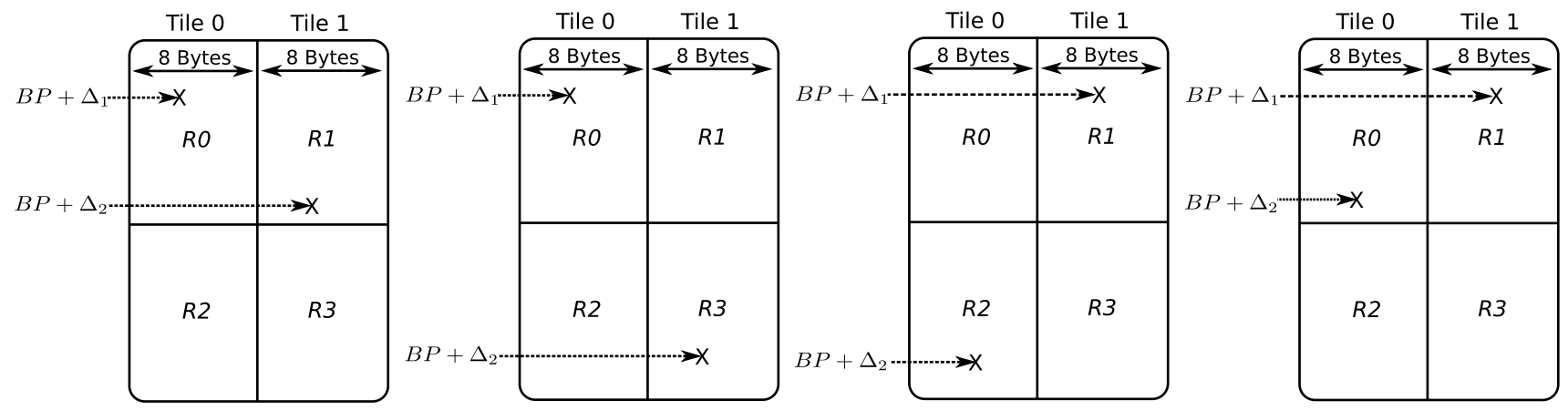

Fig. 4 Different scenarios where two memory objects belong to different tiles. BP $+\Delta$ is the address of a memory object, where BP is the base address and $\Delta$ is the offset from BP 
- Case B: $\left(\Delta_{1} \&\right.$ mask $)=\left(\Delta_{2} \&\right.$ mask $)$. In this case, memory objects of Inst 1 and Inst 2 belong to the same tile, but they may or may not belong to the same region. This can be established by calculating the absolute difference between two offsets, i.e., Diff $=a b s\left(\Delta_{1}-\Delta_{2}\right)$. An absolute difference of greater than or equal to the size of two regions (i.e., $64 \mathrm{~K}$ bytes) guarantees that the two memory objects belong to different regions in the same tile, as shown in Fig. 5a. However, there is no such guarantee when Diff is less than $64 \mathrm{~K}$ bytes. Memory objects may (e.g., Fig. 5b) or may not (e.g., Fig. 5c, d) belong to different regions in the same tile.

Since BP is not known at compile time, it is not possible to calculate region numbers for the situations, as shown in Fig. 5b-d. Therefore, we pessimistically assume that the two memory objects belonging to the same tile always belong to the same region. In other words, like Case A, we suggest appending the tile ID of a memory object as its affinity number in the affinity analysis step. In the following discussion, we break Case B into two sub-cases and compare the AAIS with the BIS in each sub-case.

- Case B1: Memory objects belong to the same tile but different regions. Table 2 compares three different BIS and AAIS-generated schedules for Case B. Let us assume that all three schedules are generated by BIS for the scenario, as depicted in Fig. 5a, b. BIS-generated instruction schedule 1 consists of one ADD instruction, two memory instructions, and an SUB instruction. With memory objects located in different regions (of the same tile), scheduling of memory instructions by BIS in the same cycle (indicated by placing $\|$ symbol between them in cycle 2) does not incur a stall. The BIS-generated schedule has an execution time of three cycles.
The corresponding AAIS-generated schedule is shown in the third column. Since memory objects belong to the same tile, the affinity analysis step pessimistically assumes that they also belong to the same region (which is not true) and appends their tile IDs as their affinity numbers. This makes the second condition to be false. As a result, AAIS serializes memory instructions by scheduling one of them in the same cycle with the ADD instruction and the other one with the SUB instruction. This reduces the execution time from 3 to 2 cycles as compared to BIS. Note that, SHAVE is a VLIW processor, capable of executing multiple instructions in the same cycle depending on the availability of functional units.

The BIS-generated instruction schedule two consists of one ADD instruction and two memory instructions. The schedule incurs no stall cycle and has an execution time of 2 cycles. As shown in the third column, AAIS serializes the two memory instructions by scheduling one of them with the ADD instruction. Although it changes the schedule as compared to BIS, the execution time remains the same as there is no change in the number of instruction cycles and stalls.

The BIS-generated instruction schedule three consists of only two memory instructions. The schedule incurs no stall cycle and has an execution time of 1 cycle. On the other hand, AAIS serializes the two memory instructions by scheduling them in different cycles. This increases the execution time from 1 to 2 cycles as compared to BIS.

In summary, AAIS in Case BI does not change the stall cycles as compared to BIS. However, it may increase, decrease, or not affect the number of instruction cycles depending on the BIS-generated instruction schedule.

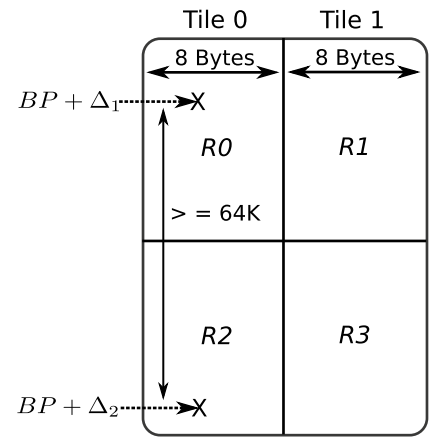

(a)

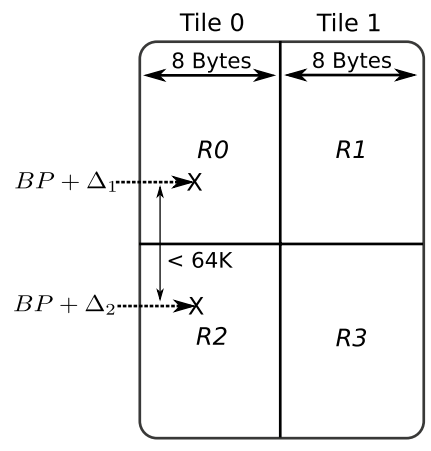

(b)
Fig. 5 Different scenarios where two memory objects belong to the same tile. Figure 5a shows that absolute difference Diff between offsets of two memory objects is greater than or equal to $64 \mathrm{~K}$ bytes, indicating that two memory objects belong to different regions. Fig-

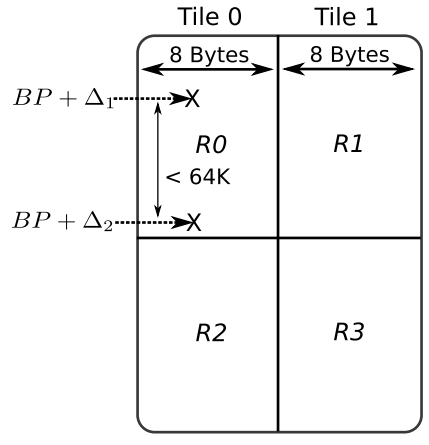

(c)

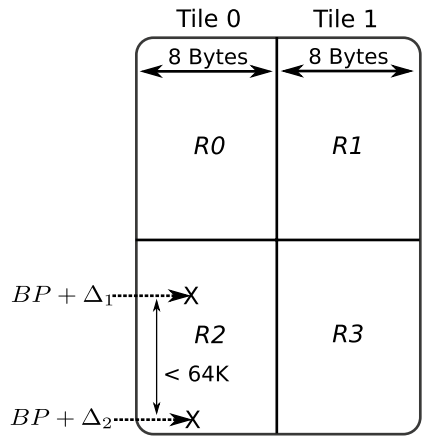

(d) ure $5 \mathrm{~b}$ shows Diff less than $64 \mathrm{~K}$ bytes and memory objects belong to different regions. Figure 5c, d shows Diff less than $64 \mathrm{~K}$ bytes, but memory objects belong to the same region 
- Case B2: Memory objects belong to the same tile and the same region. Let us assume that all BIS schedules shown in Table 2 are generated for the case, as depicted in Fig. 5c, d. With memory objects located in the same region (of the same tile), the two memory instructions scheduled in the same cycle by BIS in schedule 1 incur a stall. This results in total execution time of 4 cycles (i.e., three instruction cycles and one stall). In the corresponding AAIS-generated schedule, affinity analysis step assumes that memory objects belonging to the same tile also belong to the same region (which is true in this case). As a result, AAIS serializes memory instructions by scheduling them in different cycles (with ADD and SUB instructions). This not only avoids the stall cycle, but also reduces the instruction cycles. Hence, the execution time reduces from four to two cycles as compared to BIS.

The BIS-generated instruction schedule 2 incurs one stall cycle for two memory instructions scheduled in the same cycle. This results in an execution time of three cycles, i.e., two instruction cycles and one stall. However, AAIS serializes the two memory instructions by scheduling one of them with the ADD instruction. This saves the stall cycle and hence reduces the execution time from 3 to 2 cycles.

The BIS-generated instruction schedule 3 consists of only two memory instructions and incurs a single stall cycle, resulting in an execution time of two cycles. On the other hand, AAIS serializes the two memory instructions by scheduling them in different cycles. This avoids the stall cycle but also increase the number of instruction cycles resulting in no effect on the execution time.

In summary, AAIS in Case B2 reduces the stall cycles as compared to BIS. However, it may increase, decrease, or not affect the number of instruction cycles depending on the BIS-generated instruction schedule.

It is clear from the above discussion that AAIS can reduce stall cycles when simultaneous accesses are requested into the same region of a tile. Furthermore, above discussion shows that tile IDs can be used as affinity numbers without the loss of correctness. It is important to mention that affinity numbers are merely used as a compile time prediction for the physical location of memory objects and do not provide any means of controlling data placement.

We propose two different approaches for the first step of our solution, i.e., affinity analysis. In the source code annotation approach, tile IDs are appended to memory objects by a programmer using custom attributes. In the automated analysis approach, tile IDs are inferred from the source code by analyzing the relative addresses of memory objects. Note that any one of these two approaches can be combined with the second step (i.e., AAIS) to construct the complete solution. Both approaches are discussed in the following subsections.

\subsubsection{Source code annotation}

It is a compile time approach that involves appending the tile ID to each memory object in the application source code. This is achieved by defining a custom attribute and making the compiler aware of its syntax and semantics.

In annotation-based approach, the difference between predicted and actual physical locations of memory objects depends upon the knowledge a programmer has about the layout of application data. Tile IDs can be appended more accurately by having a good understanding of data structures used in the application and their access patterns. For example, tile IDs to be appended to array elements depend upon the location of the first element in a CMX slice (i.e., Tile 0/1), offset from the base address, and the byte alignment.

Figure 6 shows different predictions for physical locations of array elements by appending tile IDs through source code annotation. The actual placement of array elements in the CMX slice is shown in Fig. 6e. Let us assume that the $\mathrm{BC}$ simultaneously schedules the access to $\mathrm{A}[0]$ in the same cycle with $\mathrm{A}[3]$ and access to $\mathrm{A}[1]$ in the same cycle with $A[2]$. This will result in two stall cycles as two memory accesses will be blocked due to a clash between memory ports. In other words, this particular example has optimization potential of 2 . Figure $6 \mathrm{~b}$ shows the best prediction of physical locations as it reflects the real mapping of array elements in the CMX slice.

Tile0
\begin{tabular}{|c|c|c|c|}
\hline A[0] & $A[1]$ & Tile1 \\
\hline & & $A[3]$ & $A$ \\
\hline
\end{tabular}

(a) Prediction 1

Tile0
\begin{tabular}{|c|c|c|c|}
\hline & $A[0]$ & $A[1]$ & $A[2]$ \\
\hline$A[3]$ & $\vdots$ & \\
\hline
\end{tabular}

(b) Prediction 2

\begin{tabular}{l} 
Tile0 \\
\begin{tabular}{|l|l|l|}
\hline & & \multicolumn{2}{c|}{ Tile1 } \\
\hline$A[2]$ & $A[3]$ & $A[1]$ \\
\hline
\end{tabular} \\
\hline
\end{tabular}

(c) Prediction 3

Tile0
\begin{tabular}{|l|l|l|l|}
\hline & & \multicolumn{2}{c|}{ Tile1 } \\
\hline$A[1]$ & $A[2]$ & $A[3]$ & \\
\hline
\end{tabular}

(d) Prediction 4

\begin{tabular}{l} 
Tile0 \\
\begin{tabular}{|c|c|c|}
\hline & $A[0]$ & \multicolumn{2}{c|}{ Tile1 } \\
\hline$A[3]$ & & $A[2]$ \\
\hline
\end{tabular} \\
\hline
\end{tabular}

(e) Actual placement

Fig. 6 Predictions of physical locations through source code annotation 


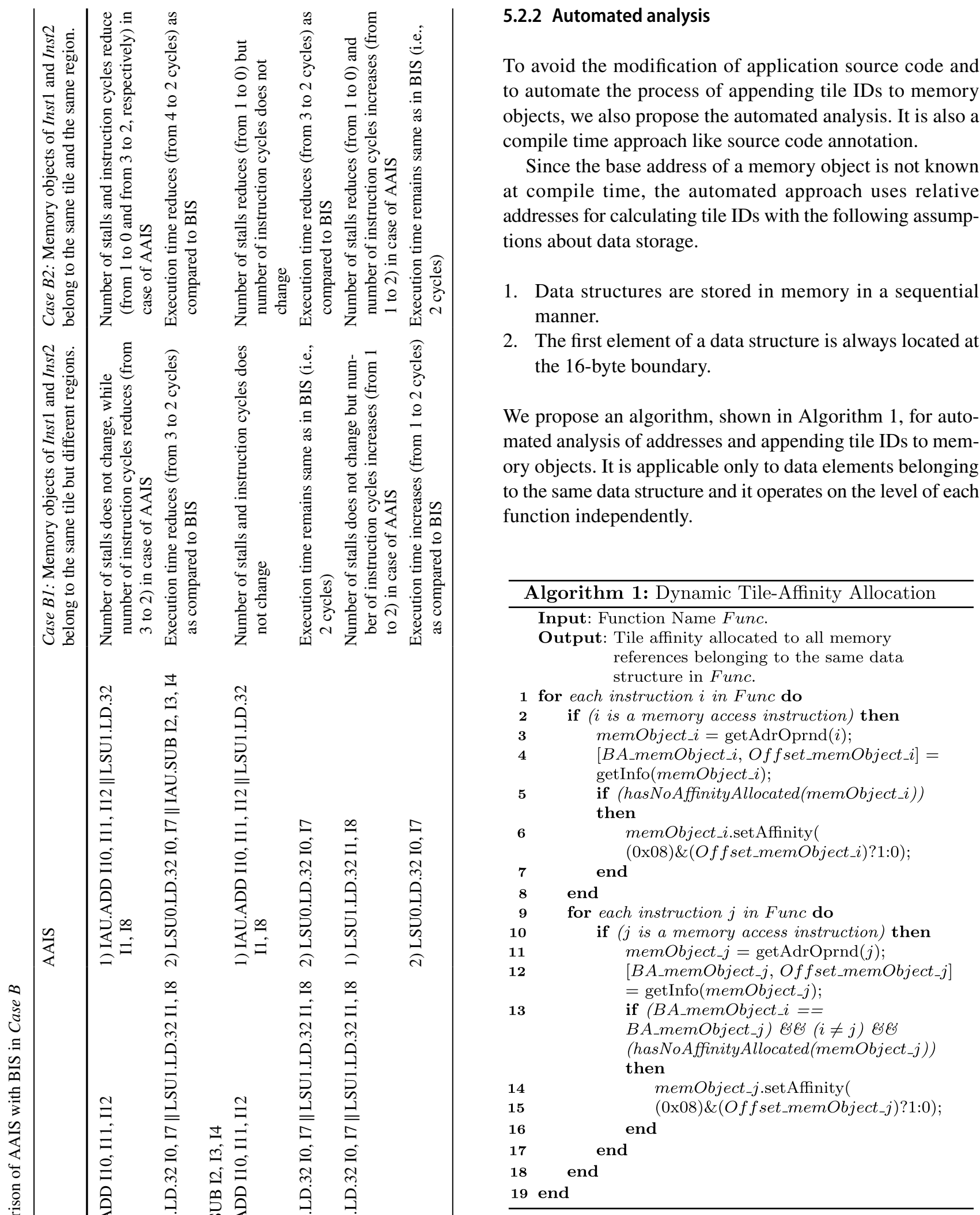

Algorithm 1 operates as follows. For each memory instruction $i$ in the function Func, the address of its memory object is retrieved into memObject_ $i$ (lines 1-3) and then broken into the base address and the offset (line 4 ). 
If not already appended with a tile ID, one is calculated for memObject $i$ (lines 5-7). Note that the width of each tile of a CMX slice is 8 bytes, as shown in Fig. 3b. Tile ID is decided based on the masking of offset value (i.e., Offset_memObject_i) with $0 \times 008$. The inner for loop (line 9-17) scans all other memory instructions in Func to find if they access a memory object with the same base address as memObject_ $i$ but with a different offset value. The condition of same base address ensures that memory objects belong to the same data structure. If the condition is true and new memory object (i.e., memObject_j) is not already appended with a tile ID, it is appended with a one based on masking of its offset value (i.e., Offset_memObject_j) with $0 \times 008$.

\subsubsection{Discussion}

AAIS leads to the reduction of memory stalls only if an application has an inherent potential for optimization. If there are no simultaneous requests for accessing the same region of a CMX slice, there will be no stall cycles in the BIS-generated schedule and hence no space for AAIS to optimize the schedule. The optimization potential of an application can be defined as the number of simultaneous memory accesses to the same region scheduled in a single cycle by BIS. Optimization potential can easily be calculated by inspecting the assembly code of an application generated by the $\mathrm{BC}$.

To harness the optimization potential of an application, physical locations of memory objects predicted by affinity numbers and their actual physical locations should be same. Otherwise, appended tile IDs will provide wrong information to the scheduler for AAIS leading to the generation of an unoptimized or unwanted instruction schedule.

The source code annotation places a burden on the programmer to attach custom attributes to memory objects. For large applications, it can be time-consuming and may also require modifications other than simply attaching custom attributes. However, if data placement can be enforced on the application data and it is a known priori, this approach could be more accurate and beneficial in reducing stall cycles than the automated one.

On the other hand, automated analysis relieves the programmer from the manual modifications in the application source code. However, it appends tile IDs to memory objects based on their offset from the base address of data structure they belong to. It also assumes that the first element of a data structure is always aligned at the 16-byte boundary. This may result in appended tile IDs (i.e., predicted physical locations) not reflecting the actual locations of memory objects.

\section{Implementation}

In this section, we provide the details of modifications applied on Myriad 2 compiler to implement the proposed solution. We first explain the implementation of affinity analysis step using both approaches, followed by the implementation details of AAIS.

\subsection{Source code annotation}

Myriad 2 compiler is an extended version of LLVM compiler framework that is tailored to generate code for a SHAVE processor. Like LLVM, Myriad 2 compiler also uses Clang as a compiler front-end for $\mathrm{C} / \mathrm{C}++$ languages. To implement the annotation-based approach, we modify the LLVM framework at two levels.

\subsubsection{Adapting the front-end}

The idea of source code annotation is to allow the programmer appending tile IDs to all memory objects in the application source code. We enable this by defining a custom attribute for any type and making Clang aware of its syntax and semantics. We modify the Clang source code as described below.

1. Add the definition of the custom attribute to Clang.

2. Modify the relevant functions in Clang to detect if a given variable declaration or initialization in the source code has the custom attribute defined in step 1 . The variable can be of any type including the pointer variable.

3. If a variable has the custom attribute, then attach the metadata to the corresponding alloc, load, or store instructions generated for allocation or initialization of the variable.

\subsubsection{Adapting the backend}

The above-mentioned modifications enable the Clang to recognize custom attribute and take the appropriate actions to process it. However, the information needs to be propagated from the front-end to the backend.

In LLVM, selection DAG builder class builds an initial directed acyclic graph providing an abstraction for code representation [50]. We modify the relevant functions in the class to propagate the predicted physical locations of memory objects down to the post-register allocation scheduling (PostRAS) pass. This is achieved by detecting the existence of metadata of the desired kind while visiting alloc, store, and load instructions. Upon finding metadata, 
tile IDs are appended to memory objects of these instructions based on the value of their metadata.

\subsection{Automated analysis}

Unlike the source code annotation, the automated analysis does not necessitate the modifications in front-end and the selection DAG builder class of the backend. We implement the automated analysis by writing a custom LLVM pass, named "Address Analysis Pass" (AAP), as shown in Fig. 7. AAP is invoked after register allocation and the generation of a basic instruction schedule. It calculates and appends tile IDs to memory objects by implementing Algorithm 1 and needs to be executed before the SHAVE PostRAS pass. Once the tile IDs are appended, SHAVE PostRAS pass creates affinity-aware instruction schedule, as described in the following subsection.

\subsection{Affinity-aware instruction scheduling (AAIS)}

In LLVM framework, the compiler consists of multiple passes which perform particular transformations and optimizations. In Myriad 2's BC, a basic instruction schedule is generated by preceding passes before AAP, as shown in Fig. 7. We modify PostRAS pass to update basic instruction schedule based on tile IDs appended to memory objects in AAP. The modified PostRAS pass detects the conflicts

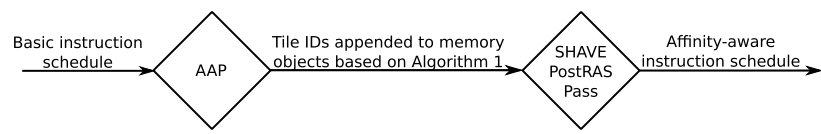

Fig. 7 Implementation of automated analysis as a custom pass in LLVM. Address analysis pass (AAP) appends affinity numbers to memory objects using Algorithm 1. Modified SHAVE PostRAS pass creates affinity-aware instruction schedule based on affinity numbers among memory instructions by comparing tile IDs of their memory objects (i.e., testing the second condition defined in Sect. 5) and saves stall cycles by not scheduling them in the same clock cycle.

\section{Experimental setup}

To evaluate our proposed optimization, we used 11 benchmarks, as described in Sect. 3. Table 3 provides a brief description of these benchmarks, while the critical part of their source code is given in Table 4 of "Appendix".

Benchmarks P1-P4 and P6 perform image addition or subtraction on different number and sizes of input images. Although a very basic operation, image addition, and subtraction is used as a step in other algorithms. Example of such algorithms includes usage of image differencing as a simple technique for change detection [51] providing a powerful interpretation of change in the tropical region and urban environment [52]. Image differencing is also used in mask mode radiography (for studying the propagation of contrast medium) and in motion-based segmentation [41]. Similarly, image addition is used in calculating the average face as a step in face recognition techniques based on eigenfaces [53]. The integral image technique is another algorithm which uses the pixel addition and is widely used in fields of computer vision and computer graphics such as texture mapping and face detection [54, 55].

Benchmark P5 and P8 perform filtering operation, while P7 represents convolution. These operations are widely used for noise reduction, sharpening, edge detection, and blurring of images [41, 56]. Benchmark P9 represents white balancing operation which is a required stage of image-processing pipeline in modern digital cameras [57, 58]. Benchmark P10 consists of histogram generation which is used as an initial step in image enhancement applications [59, 60]. Benchmark

Table 3 Brief description of benchmarks

\begin{tabular}{|c|c|c|c|c|}
\hline Prog ID & Description & $\begin{array}{l}\text { Number of } \\
\text { inputs }\end{array}$ & $\begin{array}{l}\text { Total input } \\
\text { size (KB) }\end{array}$ & Output size (KB) \\
\hline P1 & Calculates the absolute difference of two input images & 2 & 1 & 1 \\
\hline $\mathrm{P} 2$ & Performs addition of four input images & 4 & 20 & 20 \\
\hline P3 & Performs addition of two input images & 2 & 1.875 & 1.875 \\
\hline P4 & Same as P3 but performs addition of two input images based on a mask input & 3 & 0.5 & 0.5 \\
\hline P5 & Calculates the output image as the scaled addition of five box filtered input images & 5 & 1.07 & 1.07 \\
\hline P6 & Addition of two scaled images & 2 & 20 & 20 \\
\hline P7 & Image convolution using a $3 \times 3$ mask & 1 & 3.75 & 1.25 \\
\hline P8 & Sum of absolute difference using a $5 \times 5$ window & 2 & 9.375 & 1.875 \\
\hline P9 & Application of white balancing operation on a RGB image & 1 & 5.625 & 5.625 \\
\hline $\mathrm{P} 10$ & Generation of histogram for the input image & 1 & 7.56 & - \\
\hline P11 & Similarity measurement between two images & 2 & 0.625 & 4.03 \\
\hline
\end{tabular}


P11 measures the degree of similarity for a given pixel in the first image with pixels in the second image at different disparities. The similarity measurement is an important step in all stereo-matching algorithms [46, 61].

All benchmarks were executed on a Myriad 2 board with a single execution thread using both BC and AAC. For each benchmark, we measured the performance improvement as the percentage reduction in stall cycles and execution time when compared to the $\mathrm{BC}$.

\section{Evaluation}

As mentioned in Sect. 5.2.3, source code annotation is a time-consuming process and may need modifications at many places in the application source code. Therefore, we evaluate and demonstrate its working only for a single memory-intensive test program in the following subsection.

\subsection{Source code annotation for a simple memory-intensive test program}

Listing 1 shows the source code of a simple test program. The program defines an array of short type of length SIZE. The main () function calls the copyArray () function which writes to every second element of the array. For performance evaluation, stalls and instruction execution cycles are measured for the for loop of copyArray ( ) function.

A portion of assembly code generated by BC for Listing 1 is shown in Fig. 8a. In SHAVE's assembly, instructions scheduled in the same cycle are represented by placing $\|$ symbol among them. The syntax of a Store instruction is LSU $(0 \mid 1)$. STO.16 x, Y, imm, and it moves the data from the register $x$ to memory. The memory address is calculated using the content of the register $y$ as the base address and $i \mathrm{~mm}$ as the displacement.

Listing 1 Source code of test program.

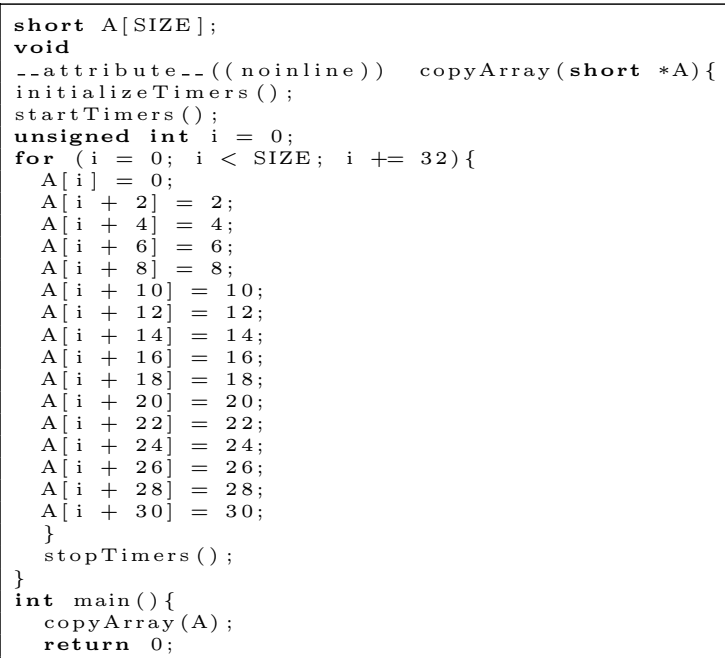

In the assembly code of Fig. 8a, i2 5 is a register containing the value of base_address +64 . Numbers on the right of the assembly code show the clock cycles in which instructions are scheduled. For ease of discussion, we assume that base_address is zero. In cycle 1 of the assembly code, two Store instructions are scheduled simultaneously. One of them accesses address $60(=0+64-4)$, located in Tile 1 and $R l$ of the CMX slice, as shown in Fig. 8b. The other paired instruction accesses the location at address 8 $(=0+64-56)$, also located in the same tile and the same region. As a result, the simultaneous scheduling of the two
//i25=base_address +64
8) LSU1.STO. 16 i9 i25 -60
II LSU0.STO.16 i10 i25 -40
7) LSU1.STO.16 i28 i25 -8
|| LSU0.STO.16 i6 i25 -64
6) LSU1.STO.16 i18 i25 - 16
II LSU0.STO.16 i29 i25 -12
5) LSU1.STO.16 i16 i25 -24
|| LSU0.STO.16 i17 i25 -20
4) LSU1.STO.16 i14 i25 -32
II LSU0.STO.16 i15 i25 -28
3) LSU1.STO.16 i $12 \mathrm{i} 25-44$
|| LSU0.STO.16 i13 i25 -36
2) LSU1.STO.16 i 1 i25 -52
|| LSU0.STO.16 i11 i25 -48
1) LSU1.STO.16 i27 i25 -4
|| LSU0.STO.16 i2 i25 -56

(a) A portion of BC-generated assembly code for Listing 1

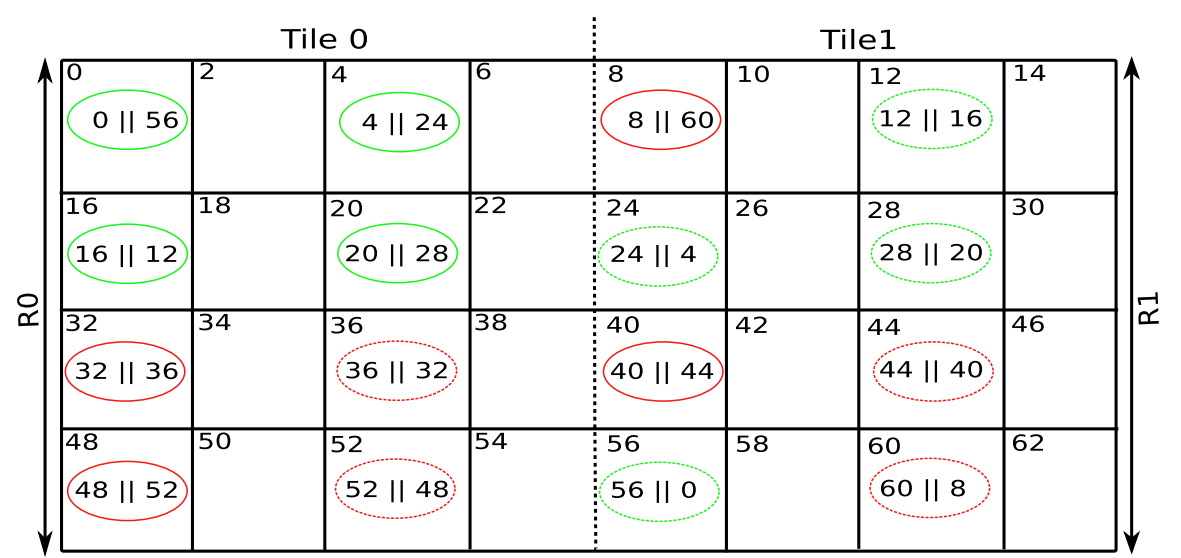

(b) Layout of relevant array elements in a CMX slice. Simultaneous memory accesses resulting in stalls are indicated by red colored ellipses

Fig. 8 Instruction schedule generated by $\mathrm{BC}$ 
instructions incurs a stall cycle, as discussed in Case B2 of section 5.2. Same is true for cycles 4, 5, and 6 .

On the other hand, the two store instructions scheduled in cycle 2 access addresses in different tiles and hence different regions. Specifically, one of them accesses the address 12 (=0+64 - 52), located in the Tile 1 and $R 1$. The other paired instruction accesses the address $16(=0+64-48)$, located in the Tile 0 and $R 0$. Therefore, simultaneous scheduling does not incur a stall cycle as discussed in Case A of section 5.2. Same is true for cycles 3, 7, and 8. Note that the optimization potential for the given piece of assembly code shown in Fig. 8a (and not for the whole program) is 4 as there are four cycles entertaining simultaneous memory accesses to the same region. The analysis of all (SIZE = 2500) memory accesses shows that the program of Listing 1 has an optimization potential of 234 cycles.

Listing 2 is functionally same as the Listing 1 , but the source code is modified to append memory objects with their tile IDs. Line 1 and 2 define two macros, Tile 0 and Tile1, using the custom defined attribute, i.e., moviAttr. As shown in lines 13-28, instead of accessing the array elements through array index, each element is accessed using a pointer carrying a tile ID of 0 or 1 .
Listing 2 Source code of test program with static affinity allocation.

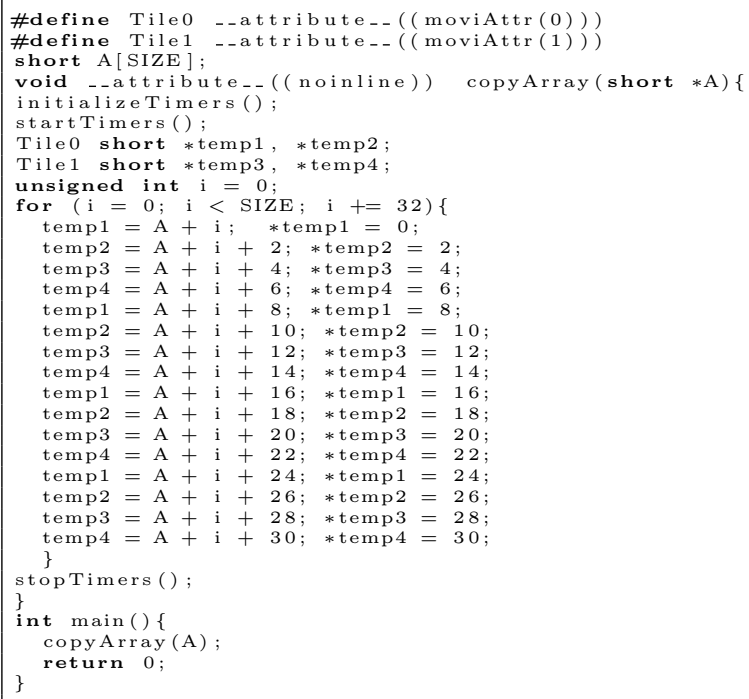

Figure 9a shows a portion of assembly code generated by AAC for the Listing 2, where register $i 18$ contains the value of base address +256 . For ease of discussion, we assume that base_address is zero. The layout of array elements in the CMX slice is shown in Fig. 9b. Assuming that the first element of the array is located at the address zero, the 16th element has an address of $32(=16 \times 2$, where 2 is the size of each element) and the 18th element has an address of 36. Since lines 21 and 22 of Listing 2 append the same tile ID to 16th and 18th array elements, AAC does not schedule accesses to them in the same cycle. As shown in Fig. 9a, address $32(=256-224)$ is accessed simultaneously with address $12(=256-244)$ in cycle 10 and both addresses are located in different tiles and hence different regions. This saves a stall cycle for accessing the 16th array
$/ /$ i18 $=$ base_address +256

13) LSU1.STO.16 i2 i18 72 II LSU0.STO.16 i6 i18 -256

12) LSU1.STO.16 i1 i18 76 II LSU0.STO.16 i9 i18 -252

11) LSU1.STO.16 i2 i18 -248 || LSU0.STO.16 il1 i18 144

10) LSU1.STO.16 i1 i18 -244 || LSU0.STO.16 i14 i18 -224

9) LSU1.STO.16 i2 i18 136

8) LSU1.STO.16 i1 i18 140 || LSU0.STO.16 i12 i18 -236 || LSU0.STO.16 i11 i18 -240

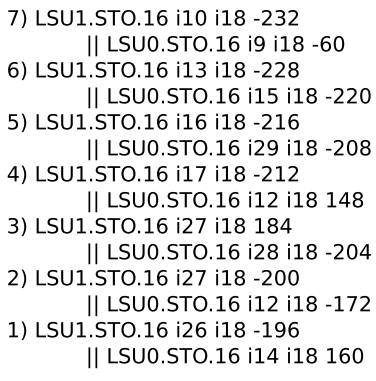

(a) A portion of AAC-generated assembly code for Listing 2

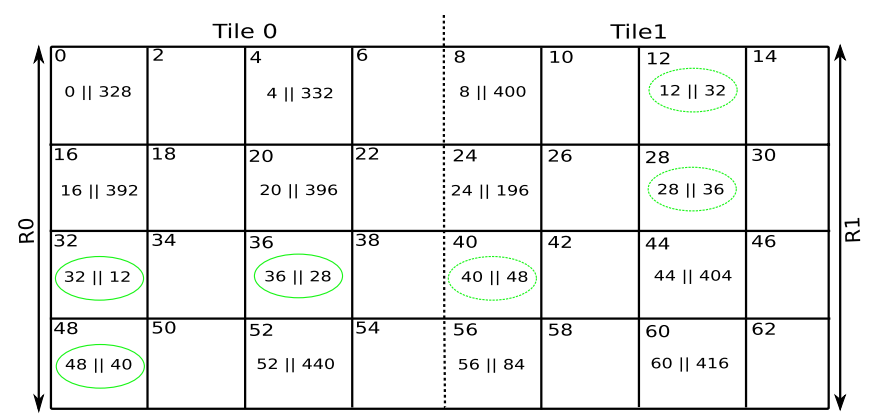

(b) Layout of relevant array elements in the CMX slice. All stalls resulting from simultaneous accesses in the same region (indicated by red colored ellipses) in the Fig. 8 are removed by AAIS

Fig. 9 Instruction scheduling with static affinity allocation for memory references 
element (located at address 32) as compared to the schedule generated by $\mathrm{BC}$, as shown in Fig. 8. In the same way, all the other stall-generating simultaneous accesses in Fig. 8 are avoided, resulting in significant reduction of stall cycles for the annotated version of the program.

Execution of the test program in Listing 1 on Myriad 2 board takes 937 clock cycles (i.e., 765 instruction cycles plus 172 stalls). On the other hand, the test program of Listing 2 takes 697 clock cycles (i.e., 641 instruction cycles plus 56 stalls). The remaining 56 stalls may belong to categories of bad speculation or core bound stalls. The difference of 240 cycles between the two execution times is very close to the optimization potential of 234 cycles for the test program in Listing 1 . The significant improvement by $25.61 \%$ in execution time of the sample test program can be attributed to its memory-intensive nature.

\subsection{Experimental results for automated affinity analysis}

In this subsection, we evaluate the classification of memory references using automated affinity analysis approach. We executed the benchmarks given in Table 3 on a Myriad 2 board using both $\mathrm{BC}$ and $\mathrm{AAC}$.

Figure 10 shows the breakdown of the execution time of benchmarks into instruction and stall cycles. The breakdown is shown for both, BC and AAC. Benchmarks executed using AAC show a significant reduction in stall cycles as compared to the BC (e.g., P1, P2, P3, P4, P6, P9, and P10). The average reduction in stall cycles is by $69.83 \%$.

Some benchmarks, such as P5 and P7, show a relatively lesser reduction in stall cycles. The difference in reduction of stall cycles across different benchmarks can be explained through their optimization potential. Figure 11 shows the breakdown of total requests for simultaneous memory access into three different cases. As explained in Sect. 5.2.3, BC and AAC generate the same schedule in Case A. In Case $B 1$, AAC can possibly reduce the number of instruction cycles. However, AAC also reduces stalls in addition to a possible reduction in the number of instruction cycles in Case B2. In other words, higher the number of simultaneous memory requests belonging to Case A lower is the Optimization Potential of a benchmark. Figure 11 shows that both benchmarks, $\mathrm{P} 5$ and $\mathrm{P} 7$, have $20 \%$ of simultaneous memory requests belonging to Case A. Therefore, AAC achieves relatively lower reduction in stall cycles for P5 and P7 as compared to other benchmarks.

For most benchmarks (i.e., P1, P3, P4, P5, P6, P7, $\mathrm{P} 9$, and $\mathrm{P} 10$ ), the number of instruction cycles remains almost same when executed using BC and AAC. However, AAC execution of $\mathrm{P} 2$ increases the number of instruction cycles by $4.83 \%$. The increase can be attributed to those

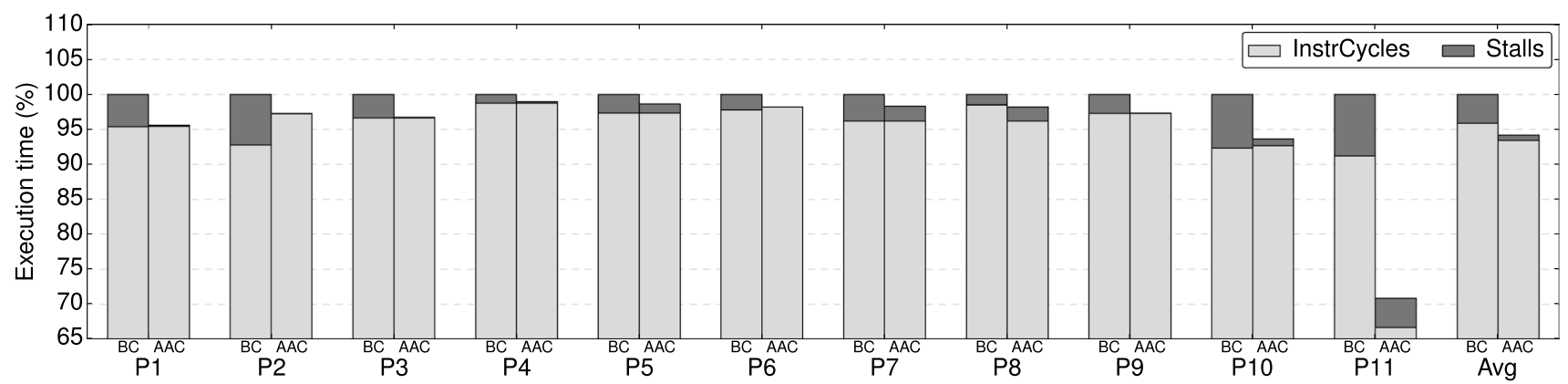

Fig. 10 Breakdown of execution time into instruction and stall cycles for executions using BC and AAC

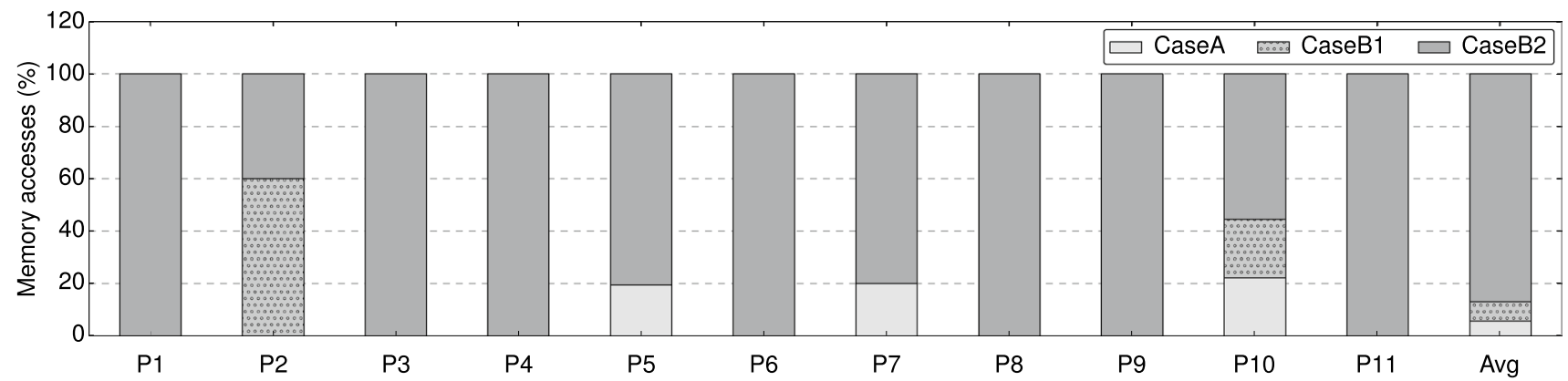

Fig. 11 Breakdown of simultaneous memory access into three different cases. Case A: Memory objects belong to different tiles. Case B: Memory object belong to the same tile but different regions. Case B_2: Memory objects belong to the same region of the same tile 
simultaneously scheduled memory instructions in the BCgenerated schedule which cannot be successfully serialized by AAC through scheduling them in the same cycle with another suitable instruction. As a result, new cycles need to be inserted for scheduling of such instructions (as explained through Schedule 3 of Table 2 in Sect. 5). On the other hand, AAC execution of P8 and P11 shows $2.34 \%$ and $24.57 \%$ reduction in instruction cycles, respectively, as compared to $\mathrm{BC}$. This reduction can be attributed to those simultaneously scheduled memory instructions in the BC-generated schedule which are successfully serialized by AAC through scheduling them in the same cycle with another suitable instruction (as explained through the Schedule 1 of Table 2 in Sect. 5).

Figure 10 shows that stall cycles make up to $8.77 \%$ (i.e., P11) of execution time and $4 \%$ on average for BC executions. Since our proposed optimization focuses only on the reduction of memory stalls, the execution time improves up to $30 \%$ (i.e., P11) with an average of $5.79 \%$. However, the significant average reduction by $69 \%$ in stall cycles suggests that the proposed optimization can substantially improve execution time for memory-intensive applications, as shown by a sample program in Sect. 8.1.

\section{Conclusion}

In this paper, we propose the classification of memory references as a compiler optimization to reduce the memory bound stalls incurred by an application running on a visionprocessing system (i.e., Myriad 2 MPSoC). Our solution consists of two steps: affinity analysis and affinity-aware instruction scheduling. We implemented two different approaches for affinity analysis, namely, source code annotation and automated analysis.

While source code annotation approach facilitates more accurate prediction of the physical location of memory objects, it needs considerable effort by the programmer to modify the source code. On the other hand, automated analysis relieves a programmer from the burden of modifying the source code, but employs certain assumptions about data placement. This may result with less accuracy in attaching affinity numbers to memory objects.

Experimental results show that by making the compiler aware of memory architecture and efficiently using the dual load-store units, memory stalls can be reduced significantly. Classification of memory references using source code annotation reduces stalls by $67.44 \%$ for a memory-intensive program, leading to $25.61 \%$ improvement in its execution time. On the other hand, automated analysis approach shows an average reduction by $69.83 \%$ in stall cycles with a modest improvement by $5.79 \%$ in execution time over a set of eleven different image-processing benchmarks.

Acknowledgements This work is supported by European Union's Horizon2020 research and innovation programme under grant agreement number 687698 and Ph.D. scholarship from Higher Education Commission (HEC) of Pakistan awarded to Naveed Ul Mustafa.

\section{Appendix: A critical part of source code for benchmarks}

See Table 4 


\section{References}

1. Pulli, K., Baksheev, A., Kornyakov, K., Eruhimov, V.: Real-time computer vision with opencv. Commun. ACM 55(6), 61-69 (2012)

2. Farabet, C., Martini, B., Corda, B., Akselrod, P., Culurciello, E., LeCun, Y.: Neuflow: a runtime reconfigurable dataflow processor for vision. In: Proceedings of IEEE Computer Society Conference on Computer Vision and Pattern Recognition Workshops (CVPRW), pp. 109-116 (2011)

3. Barry, B., Brick, C., Connor, F., Donohoe, D., Moloney, D., Richmond, R., O'Riordan, M.J., Toma, V.: Always-on vision processing unit for mobile applications. IEEE Micro. 35(2), 56-66 (2015)

4. Chua, J.L., Chang, Y.C., Lim, W.K.: A simple vision-based fall detection technique for indoor video surveillance. Signal Image Video Process. 9(3), 623-633 (2015)

5. Gómez, M.J., García, F., Martín, D., de la Escalera, A., Armingol, J.M.: Intelligent surveillance of indoor environments based on computer vision and 3D point cloud fusion. Exp. Syst. Appl. 42(21), 8156-8171 (2015)

6. Rautaray, S.S., Agrawal, A.: Vision based hand gesture recognition for human computer interaction: a survey. Artif. Intell. Rev. 43(1), 1-54 (2015)

7. Suwajanakorn, S., Kemelmacher-Shlizerman, I., Seitz, S.M.: Total moving face reconstruction. In: Proceedings of European Conference on Computer Vision, pp. 796-812 (2014)

8. Smolyanskiy, N., Huitema, C., Liang, L., Anderson, S.E.: Realtime 3D face tracking based on active appearance model constrained by depth data. Image Vis. Comput. 32(11), 860-869 (2014)

9. Bar. Y., Diamant, I., Wolf, L., Greenspan, H.: Deep learning with non-medical training used for chest pathology identification. In: Proceedings of Medical Imaging 2015: Computer-Aided Diagnosis (2015)

10. Greenspan, H., van Ginneken, B., Summers, R.M.: Guest editorial deep learning in medical imaging: overview and future promise of an exciting new technique. IEEE Trans. Med. Imaging. 35(5), 1153-1159 (2016)

11. Ohn-Bar, E., Tawari, A., Martin, S., Trivedi, M.M.: On surveillance for safety critical events: in-vehicle video networks for predictive driver assistance systems. Comput. Vis. Image Underst. 134, 130-140 (2015)

12. Mandal, D.K., Sankaran, J., Gupta, A., Castille, K., Gondkar, S., Kamath, S., Sundar, P., Phipps, A.: An Embedded Vision Engine (EVE) for automotive vision processing. In: Proceedings of IEEE International Symposium on Circuits and Systems (ISCAS), pp. 49-52 (2014)

13. Zhang, B., Huang, W., Li, J., Zhao, C., Fan, S., Wu, J., Liu, C.: Principles, developments and applications of computer vision for external quality inspection of fruits and vegetables: a review. Food Res. Int. 62, 326-343 (2014)

14. Aghbashlo, M., Hosseinpour, S., Ghasemi-Varnamkhasti, M.: Computer vision technology for real-time food quality assurance during drying process. Trends Food Sci. Technol. 39(1), 76-84 (2014)

15. Ma, J., Sun, D.W., Qu, J.H., Liu, D., Pu, H., Gao, W.H., Zeng, X.A.: Applications of computer vision for assessing quality of agri-food products: a review of recent research advances. Crit. Rev. Food Sci. Nutr. 56(1), 113-127 (2016)

16. Guo, Y., Zhuge, Q., Hu, J., Yi, J., Qiu, M., Sha, E.H.M.: Data placement and duplication for embedded multicore systems with scratch pad memory. IEEE Trans. Comput. Aided Des. Integr. Circuits Syst. 32(6), 809-817 (2013)

17. Wang, D., Du, X., Yin, L., Lin, C., Ma, H., Ren, W., Wang, H., Wang, X., Xie, S., Wang, L., Liu. Z., Wang, T., Pu, Z., Ding, G.,
Zhu, M., Yang, L., Guo, R., Zhang, Z., Lin, X., Hao, J., Yang, Y., Sun, W., Zhou, F., Xiao, N., Cui, Q., Wangg, X.: MaPU: A novel mathematical computing architecture. In: Proceedings of IEEE International Symposium on High Performance Computer Architecture (HPCA), pp. 457-468 (2016)

18. Lin, Z., Sankaran, J., Flanagan, T.: Empowering automotive vision with TI's Vision AccelerationPac. TI White Paper (2013)

19. Conti, F., Rossi, D., Pullini, A., Loi, I., Benini, L.: PULP: a ultralow power parallel accelerator for energy-efficient and flexible embedded vision. J. Signal Process. Syst. 84(3), 339-354 (2016)

20. Machine Vision Technology: Movidius https://www.movidius. com/technology. Accessed 23 Sept 2017

21. Diken, E., O'Riordan, M.J., Jordans, R., Jozwiak, L., Corporaal, H., Moloney, D.: Mixed-length simd code generation for vliw architectures with multiple native vector-widths. In: Proceedings of IEEE 26th International Conference on Application-specific Systems, Architectures and Processors (ASAP), pp. 181-188 (2015)

22. Chen, T.P., Budnikov, D., Hughes, C.J, Chen, Y.K.: Computer vision on multi-core processors: articulated body tracking. In: Proceedings of IEEE International Conference on Multimedia and Expo, pp. 1862-1865 (2007)

23. Lattner, C., Adve, V.: LLVM: A compilation framework for lifelong program analysis \& transformation. In: Proceedings of Second Annual IEEE/ACM International Symposium on Code Generation and Optimization (CGO), pp. 75-88 (2004)

24. Sethia, A., Dasika, G., Mudge, T., Mahlke, S.A.: Customized processor for energy efficient scientific computing. IEEE Trans. Comput. 61(12), 1711-1723 (2012)

25. Cho, J., Paek, Y., Whalley, D.: Efficient register and memory assignment for non-orthogonal architectures via graph coloring and MST algorithms. In: Proceedings of the Joint Conference on Languages, Compilers and Tools for Embedded Systems: Software and Compilers for Embedded Systems (LCTES/SCOPES), pp. 130-138 (2002)

26. Leupers, R., Kotte, D.: Variable partitioning for dual memory bank DSPs. In: Proceedings of IEEE International Conference on Acoustics, Speech, and Signal Processing (ICASSP), pp. 1121$1124(2001)$

27. Ko, M.Y., Bhattacharyya, S.S.: Partitioning for DSP software synthesis. In: Proceedings of International Workshop on Software and Compilers for Embedded Systems (SCOPES), pp. 344-358 (2003)

28. Murray, A., Franke, B.: Fast source-level data assignment to dual memory banks. In: Proceedings of the 11th International Workshop on Software and Compilers for Embedded Systems (SCOPES), pp. 43-52 (2008)

29. Sipkova, V.: Efficient variable allocation to dual memory banks of DSPs. In: Proceedings of International Workshop on Software and Compilers for Embedded Systems (SCOPES), pp. 359-372 (2003)

30. Kim, Y., Lee, J., Shrivastava, A., Paek, Y.: Operation and data mapping for CGRAs with multi-bank memory. In: Proceedings of the ACM SIGPLAN/SIGBED Conference on Languages, Compilers, and Tools for Embedded Systems (LCTES), pp. $17-26(2010)$

31. Mi, W., Feng, X., Xue, J., Jia. Y.: Software-hardware cooperative DRAM bank partitioning for chip multiprocessors. In: Proceedings of International Conference on Network and Parallel Computing (IFIP), pp. 329-343 (2010)

32. Bircsak, J., Craig, P., Crowell, R., Cvetanovic, Z., Harris, J., Nelson, C.A, Offner, C.D.: Extending openmp for NUMA machines. In: Proceedings of ACM/IEEE 2000 Conference on Supercomputing (SC) (2000)

33. Antony, J., Janes, P.P., Rendell, A.P.: Exploring thread and memory placement on numa architectures: Solaris and linux, ultrasparc/fireplane and opteron/hypertransport. In: Proceedings of 
International Conference on High-Performance Computing, pp. 338-352 (2006)

34. Lameter, C.: Numa (non-uniform memory access): an overview. ACM Queue. 11(7), 1-12 (2013)

35. Ribeiro, C.P., Mehaut, J.F., Carissimi, A., Castro, M., Fernandes, L.G.: Memory affinity for hierarchical shared memory multiprocessors. In: Proceedings of 21st International Symposium on Computer Architecture and High Performance Computing, pp. 59-66 (2009)

36. Kleen, A.: A numa api for linux. SUSE Labs (2004). http://halob ates.de/numaapi3.pdf. Accessed 23 Sept 2017

37. Löf, H.,Holmgren, S.: Affinity-on-next-touch: increasing the performance of an industrial pde solver on a cc-numa system. In: Proceedings of 19th Annual International Conference on Supercomputing (SC), pp. 387-392 (2005)

38. Lankes, S., Bierbaum, B., Bemmerl, T.: Affinity-on-next-touch: an extension to the linux kernel for numa architectures. In: Proceedings of International Conference on Parallel Processing and Applied Mathematics, pp. 576-585 (2010)

39. Golgin, B., Furmento, N.: Enabling high-performance memory migration for multithreaded applications on LINUX. In: Proceedings of IEEE International Symposium on Parallel \& Distributed Processing (IPDPS) (2009)

40. Codrescu, L., Anderson, W., Venkumanhanti, S., Zeng, M., Plondke, E., Koob, C., Ingle, A., Tabony, C., Maule, R.: Hexagon DSP: an architecture optimized for mobile multimedia and communications. IEEE Micro. 34(2), 34-43 (2014)

41. Gonzalez, R.C.: Digital Image Processing. Prentice-Hall, Upper Sadle River (2002)

42. McDonnell, M.J.: Box-filtering techniques. Comput. Graph. Image Process. 17(1), 65-70 (1981)

43. Podlozhnyuk, V.: Image convolution with cuda. NVIDIA Corporation white paper, vol 2097(3), (2007)

44. Niitsuma, H., Maruyama, T.: Sum of absolute difference implementations for image processing on fpgas. In: Proceedings of International Conference on Field Programmable Logic and Applications (FPL), pp. 167-170 (2010)

45. Bianco, S., Gasparini, F., Schettini, R.: Combining strategies for white balance. In: Proceedings of SPIE 6502, Digital Photography III, pp. 65020D (2007)

46. Hirschmuller, H., Scharstein, D.: Evaluation of cost functions for stereo matching. In: Proceedings of IEEE Conference on Computer Vision and Pattern Recognition (CVPR), pp. 1-8 (2007)

47. Reinders, J.: VTune Performance Analyzer Essentials. Intel Press, Santa Clara (2005)

48. Moloney, D., Barry, B., Richmond, R., Connor, F., Brick, C., Donohoe, D.: Myriad 2: Eye of the computational vision storm. In: Proceedings of Hot Chips 26 Symposium (HCS), pp. 1-18 (2014)

49. Thorarensen, S.: A back-end for the skepu skeleton programming library targeting the low-power multicore vision processor myriad 2. Master's thesis, Linköping university, Sweden (2016)

50. LLVM 6 documentation https://llvm.org/docs/CodeGenerator. html\#introduction-to-selectiondags. Accessed 23 Sept 2017

51. Hussain, M., Chen, D., Cheng, A., Wei, H., Stanley, D.: Change detection from remotely sensed images: from pixel-based to object-based approaches. ISPRS J. Photogramm. Remote Sens. 80, 91-106 (2013)
52. S, M., Shetty, A.: A comparative study of image change detection algorithms in MATLAB. In: Proceedings of International Conference on Water Resources, Coastal and Ocean Engineering (ICWRCOE) pp. 1366-1373 (2015)

53. Turk, M., Pentland, A.: Eigenfaces for recognition. J Cogn Neurosci. 3(1), 71-86 (1991)

54. Crow, F.C.: Summed-area tables for texture mapping. In: Proceedings of 11th International Conference on Computer Graphics and Interactive Techniques pp. 207-212 (1984)

55. Jiang, L., Xie, H., Pan, B.: Speeding up digital image correlation computation using the integral image technique. Opt. Lasers Eng. 65, 117-122 (2015)

56. He, K., Sun, J., Tang, X.: Guided image filtering. IEEE Trans. Pattern Anal. Mach. Intell. 35(6), 1397-1409 (2013)

57. Ramanath, R., Snyder, W.E., Yoo, Y., Drew, M.S.: Color image processing pipeline. IEEE Signal Process. Mag. 22(1), 34-43 (2005)

58. Lukac, R.: New framework for automatic white balancing of digital camera images. Signal Process. 88(3), 582-593 (2008)

59. Arici, T., Dikbas, S., Altunbasak, Y.: A histogram modification framework and its application for image contrast enhancement. IEEE Trans. Image Process. 18(9), 1921-1935 (2009)

60. Duan, J., Qiu, G.: Novel histogram processing for colour image enhancement. In: Proceedings of Third International Conference on Image and Graphics (ICIG) pp. 55-58 (2004)

61. Hong, W.: A study of fast, robust stereo-matching algorithms. Doctoral dissertation, Massachusetts Institute of Technology, USA, (2010)

Naveed UI Mustafa is a Ph.D. student at department of Computer Engineering, Bilkent University, Ankara, Turkey. He received his M.S. degree from Royal Institute of Technology (KTH), Stockholm, in 2011. His research interests include compiler optimizations, software fault tolerance, network on chip (NoC) and real time operating systems. He can be reached at naveed.mustafa@bilkent.edu.tr.

Martin J. O'Riordan is the chief compiler architect at Movidius, where he leads the SHAVE processor compiler development based on LLVM infrastructure and also worked on the SHAVE instruction set architecture and Myriad microarchitecture. O'Riordan has a BSc in computer science from Trinity College Dublin. He can be reached at martin. oriordan@movidius.com.

Stephen Rogers is a member of the SHAVE processor compiler development team at Movidius. Contact him at stephen.rogers@movidius. com.

Ozcan Ozturk is an Associate Professor in the Department of Computer Engineering at Bilkent University. His research interests are in the areas of accelerators, manycore architectures, parallel computing, and computer architecture. Prior to joining Bilkent, he worked in Cellular and Handheld Group at Intel and Marvell. He also held positions in NEC Labs and Arizona State University. His research has been recognized by Fulbright, Turk Telekom, IBM, Intel, HiPEAC, Tubitak, and European Commission. Contact him at ozturk@cs.bilkent.edu.tr. 